\title{
Comparaison de l'impact radiologique de la production d'électricité par des réacteurs à eau sous pression ou par des réacteurs de fusion du type Tokamak
}

\author{
F. RANCILLAC *, A. DESPRES*
}

(Manuscrit reçu le 23 janvier 1990)

RÉSUMÉ Dans cet article on compare l'impact de l'ensemble du cycle du combustible nucléaire correspondant aux réacteurs à eau légère avec celui de l'ensemble du cycle du combustible associé au futur réacteur de fusion, utilisant comme source d'énergie des réactions de fusion tritium-deutérium. La comparaison a été faite sur une base de production d'énergie de $1 \mathrm{GWe}$.an. Les critères de comparaison choisis ont été les inventaires de produits combustibles manipulés, les rejets radioactifs effectués sur l'ensemble des installations, les engagements d'équivalents de dose efficace délivrés à la population et le volume des déchets. Le risque accidentel n'a pas été pris en compte.

Pour les réacteurs de fusion, quelques incertitudes résident encore qui ne permettent pas de connaître précisément la quantité de produits autres que le tritium rejeté au niveau du réacteur. Seuls des ordres de grandeur extrapolés du Tokamak NET sont donnés. Malgré ces incertitudes, il semblerait plus intéressant, du point de vue des rejets et des doses, de produire de l'électricité par des réacteurs de fusion, bien que cette filière ne supprime pas tous les problèmes relatifs aux rejets, à la manipulation et au stockage à plus ou moins long terme de produits radioactifs. Cette filière conduit également à des déchets de haute activité, auxquels sont associés des débits d'irradiation plus faibles à long terme que ceux des déchets de la filière des réacteurs à eau pressurisée.

ABSTRACT The impacts of respectively light water reactors and a planned fusion reactor, for which tritium-deuterium fusion reactions will act as energy source, have been compared. The comparison is based on a generated capacity of 1 GWe.y, using the following criteria : fuel inventories, radioactive releases, collective effective dose equivalent commitments to the public and the volume of waste. The accidental risk is not introduced.

Fusion reactor parameters are still subject to uncertainties, which prevent accurate quantification of radionuclide releases (tritium apart) from the nuclear plant. Only orders of magnitude extrapolated from values for the NET Tokamak are given. Despite these uncertainties, it would seem more interesting, from the dosimetric point of view, to use fusion reactors to produce electricity, although problems of radioactive releases, handling and long-term storage of radioactive waste would remain. Fusion reactors also generate high-level wastes with long-term exposure rates that are lower than those of light water reactors.

* Commissariat à l'énergie atomique, IPSN, Service d'études générales de protection, BP 6, 92265 Fontenay-aux-Roses Cedex. 


\section{INTRODUCTION}

L'énergie produite par les réacteurs de fusion (RF) est-elle réellement plus "propre" que celle produite par les réacteurs à eau ordinaire sous pression (REP) ? Même si les machines produisant des réactions de fusion ou Tokamaks n'en sont encore qu'au stade expérimental, les caractéristiques du premier réacteur de fusion se précisent.

La comparaison faite ici porte sur l'ensemble des cycles du combustible associé à un REP et à un RF en prenant pour critères les rejets, les équivalents de dose efficace collectifs délivrés à la population et le volume des déchets et leur activité. Afin de faciliter la comparaison, les critères sont rapportés à une production de 1 GWe.an.

L'exposition professionnelle n'est pas prise en compte ici, car les tâches à accomplir par les travailleurs dans les RF ne sont pas encore suffisamment bien définies. Les rejets accidentels ne sont pas considérés.

\section{CYCLE DU COMBUSTIBLE NUCLÉAIRE POUR PRODUIRE DE L'ÉLECTRICITÉ PAR FISSION}

\section{a. Opérations sur le combustible et inventaire}

Deux cents tonnes d'uranium naturel sont nécessaires pour produire 1 GWe.an. Or, le minerai ne contient que quelques pour mille à quelques pour cent d'uranium (sous forme d' $\mathrm{u}_{3} \mathrm{O}_{8}$ ). La première opération, après l'extraction, est donc le raffinage du minerai. A l'issue du raffinage, qui consiste en un tri mécanique, un broyage et un traitement chimique, on dispose d'un concentré, contenant de 60 à $70 \%$ d'uranium, appelé "yellow cake". Ce concentré est ensuite purifié par voie chimique, puis transformé en tétrafluorure d'uranium $\left(\mathrm{UF}_{4}\right)$.

L'uranium, pour être utilisé dans les réacteurs à eau légère, la filière de loin la plus répandue en France et dans le monde, doit passer d'une concentration en ${ }^{235} \mathrm{U}$ (isotope fissile) de $0,7 \%$ (teneur de l'uranium naturel) à une teneur comprise entre 2 et $4 \%$. Cette opération, dite enrichissement, nécessite préalablement la transformation de l'uranium en hexafluorure $\left(U_{6}\right)$ sous forme gazeuse. Le sous-produit de cette étape est une grande quantité d'uranium appauvri (teneur en ${ }^{235} \mathrm{U}$ de l'ordre de $0,2 \%$ ), qui peut être utilisée dans les réacteurs surgénérateurs.

L'UF $_{6}$ enrichi, qui se présente sous forme solide, est gazéifié, puis

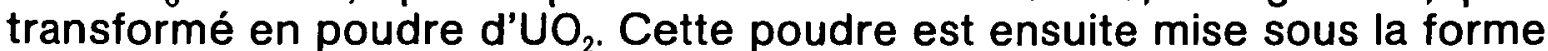
de pastilles d' $\mathrm{UO}_{2}$. Lors des opérations de fabrication du combustible, l'uranium est à l'état solide. II s'ensuit que les rejets atmosphériques sont pratiquement inexistants à ce stade. 
Le temps de séjour du combustible dans le réacteur est d'environ trois ans, à l'issue desquels il est retiré du cœur et stocké en piscine pour refroidissement. Deux options sont ensuite possibles. Les éléments combustibles irradiés sont stockés en tant que déchets (cycle ouvert), ou alors ils sont retraités (cycle fermé). Cette opération consiste à séparer, actuellement par des processus chimiques, d'une part l'uranium non utilisé et le plutonium produit, qui sont des matériaux nobles récupérables, et d'autre part les produits de fission et les actinides autres que le plutonium, qui constituent les déchets. L'uranium et le plutonium sont réinjectés dans le cycle. Le plutonium, pour sa part, peut être utilisé dans les réacteurs à eau légère sous forme de combustible MOX (mixed oxyde fuel) et dans les réacteurs surgénérateurs [7].

b. Les rejets (tableau I)

TABLEAU I

Estimation des rejets gazeux et liquides des différentes phases du cycle du combustible fission

\begin{tabular}{|c|c|c|c|}
\hline $\begin{array}{l}\text { Mines } \\
+ \text { usine } \\
+ \text { terrils }\end{array}$ & $\begin{array}{l}{ }^{238} \mathrm{U}+{ }^{234} \mathrm{U}+{ }^{230} \mathrm{Th} \\
{ }^{226} \mathrm{Ra}+{ }^{210} \mathrm{~Pb}+{ }^{210} \mathrm{Po} \\
{ }^{222} \mathrm{Rn} \\
\mathrm{U} \text { nat }\end{array}$ & $\begin{array}{l}\text { Atmosphériques } \\
\text { Liquides }\end{array}$ & $\begin{array}{c}1,575 \\
21880^{1,575} \\
25 \\
\end{array}$ \\
\hline $\begin{array}{l}\text { Traitement } \\
\text { du minerai } \\
\text { Conversion } \\
\text { Enrichissement }\end{array}$ & $\begin{array}{l}{ }^{238} \mathrm{U} \\
238 \mathrm{U} \\
238 \mathrm{U} \\
{ }^{238} \mathrm{U}\end{array}$ & $\begin{array}{l}\text { Atmosphériques } \\
\text { Liquides } \\
\text { Atmosphériques } \\
\text { Liquides }\end{array}$ & $\begin{array}{l}0,006 \\
0,008 \\
0,0025 \\
0,00005\end{array}$ \\
\hline $\begin{array}{l}\text { Fabrication du } \\
\text { combustible }\end{array}$ & ${ }^{238} \mathrm{U}$ & $\begin{array}{l}\text { Atmosphériques } \\
\text { Liquides }\end{array}$ & $\begin{array}{l}0,0065 \\
0,03\end{array}$ \\
\hline $\begin{array}{l}\text { Irradiation du } \\
\text { combustible }\end{array}$ & $\begin{array}{l}\text { Gaz rare } \\
\mathrm{T} \\
{ }^{133} \mathrm{I},{ }^{131} \mathrm{I} \\
{ }^{14} \mathrm{C} \\
\text { Particules } \\
T \\
\text { Autres }\end{array}$ & Atmosphériques & $\begin{array}{r}225000 \\
5900 \\
5,6 \\
300 \\
4,5 \\
27400 \\
15 \\
\end{array}$ \\
\hline Retraitement & $\begin{array}{l}{ }^{\mathrm{T}} \\
{ }^{85} \mathrm{Kr} \\
\text { Aérosols alpha } \\
\text { Aérosols bêta } \\
\mathrm{T} \\
\text { Total béta } \\
{ }^{106} \mathrm{Ru} \\
\text { Total alpha }\end{array}$ & $\begin{array}{l}\text { Atmosphériques } \\
\text { Liquides }\end{array}$ & $\begin{array}{c}1800 \\
100000 \\
0,002 \\
0,02 \\
260000 \\
210000 \\
143000 \\
90\end{array}$ \\
\hline
\end{tabular}


A chaque étape du cycle, les différentes opérations impliquent des rejets, dans l'air ou dans l'eau, d'isotopes radioactifs. En amont du réacteur, il ne s'agit que de radioéléments naturels; les radioéléments artificiels, produits de fission et d'activation, n'apparaissent qu'au niveau des réacteurs, des usines de retraitement, et des stockages de déchets.

L'extraction des mines provoque essentiellement l'émission atmosphérique de ${ }^{222} \mathrm{Rn}$ avec ses produits de filiation. Les terrils dégagent aussi ces mêmes produits mais les mesures prises (arrosage, recouvrement avec de la terre) conduisent à limiter les rejets. Du ${ }^{226} \mathrm{Ra}$ est également évacué avec les eaux d'exhaure et les eaux de traitement chimique du minerai, mais la majeure partie sédimente dans les bassins de rétention.

Au stade de la fabrication du combustible, l'uranium est manipulé à l'état solide, si bien que les rejets sont négligeables.

Au niveau du réacteur, l'activation des matériaux de structure, la diffusion des produits de fission à travers les différentes barrières ainsi que l'existence de quelques fuites au niveau des crayons de combustible conduisent, après traitement, au rejet d'isotopes radioactifs. Dans l'air, ces rejets sont essentiellement constitués de gaz rares et de tritium. Le tritium est également rejeté par voie liquide, ainsi qu'une faible quantité de produits solides.

Au niveau du retraitement du combustible, les gaz rares, le carbone et le tritium constituent l'essentiel des rejets atmosphériques. Dans les rejets liquides, on retrouve la plupart des produits de fission et des produits d'activation, ainsi que quelques éléments transuraniens.

L'essentiel de l'activité rejetée liée à l'ensemble du cycle du combustible est principalement dû au retraitement du combustible $(87 \%)$, suivi ensuite par les rejets des réacteurs (12 \%). Pour produire 1 GWe.an avec un REP, 1,6 Bq d'uranium seront émis dans l'atmosphère et $25 \mathrm{GBq}$ dans les eaux de surfaces. Au total, seront également rejetés pour l'ensemble du cycle du combustible $295 \mathrm{TBq}$ de tritium, $21,9 \mathrm{TBq}$ de radon 222 [7].

\section{c. Les déchets}

II y a lieu de distinguer deux types de déchets: faible et moyenne activité et haute activité. Les déchets de faible activité comprennent:les déchets provenant de l'exploitation des réacteurs et la majorité (en volume) des déchets issus du retraitement. Les déchets de moyenne et haute activité issus du retraitement sont, à l'heure actuelle, disposés dans des stockages provisoires.

Les installations de stockage sont conçues pour que les eaux d'infiltration soient récupérées. II n'y a donc normalement pas de rejet à ce stade. Les seules possibilités pouvant conduire à des rejets sont liées à une éventuelle détérioration du site. Pour les sites de stockage de déchets de faible et moyenne activité, on prévoit de surveiller le site et 
de l'entretenir pendant 300 ans après fermeture du site. Des études sont menées pour définir des sites pouvant accueillir définitivement les déchets de haute activité.

Au total, en tenant compte du retraitement du combustible, 36 à $57 \mathrm{~m}^{3}$ de déchets haute activité sont produits par an, avec une activité de 6 à $11.10^{6} \mathrm{TBq}$ pour une production de 1 GWe.an [7]. Les terrils ne sont pas comptabilisés ici, de même que les déchets de démantèlement des REP ou des usines de retraitement ou de conversion, et d'enrichissement.

\section{d. Les Joses}

L'ensemble des engagements d'équivalents de dose efficace distribués à la population du fait des opérations liées au cycle du combustible est donné dans le tableau II.

TABLEAU $\|$

Bilan concernant la production d'énergie par fission pour 1 GWe.an

\begin{tabular}{|c|c|c|}
\hline Extraction (radon) & $U$ nat & 2,0 \\
\hline $\begin{array}{l}\text { Traitement (sauf terrils) } \\
\text { U, Th, Ra } \\
\text { Rn }\end{array}$ & $200 \mathrm{t}$ & $\begin{array}{l}0,06 \\
0,1\end{array}$ \\
\hline Conversion et enrichissement & $\mathrm{U} 155 \mathrm{t}$ & 0,03 \\
\hline \multicolumn{2}{|l|}{$\begin{array}{l}\text { Fabrication du combustible } \\
\text { REP }\end{array}$} & 0,0007 \\
\hline $\begin{array}{l}\text { Réacteur } \\
\text { a) rejets atmosphériques } \\
\text { b) rejets liquides }\end{array}$ & $\begin{array}{l}\mathrm{U} 80 \mathrm{t} \\
\mathrm{Pu} 300 \mathrm{~kg}\end{array}$ & $\begin{array}{l}0,74 \\
0,001-0,025 \text { (mer) } \\
0,004 \text { (rivière) }\end{array}$ \\
\hline $\begin{array}{l}\text { Retraitement } \\
\text { a) rejets atmosphériques } \\
\text { b) rejets liquides }\end{array}$ & $\begin{array}{l}\mathrm{U} 27 \mathrm{t} \\
\mathrm{Pu} 260 \mathrm{~kg}\end{array}$ & $\begin{array}{l}0,5 * \\
12,0 *\end{array}$ \\
\hline Déchets haute activité & environ 50 & \\
\hline
\end{tabular}

- Ces valeurs considèrent que $100 \%$ du combustible est retraité, dans la réalité d'aujourd'hui seul $5 \%$ du combustible est retraité.

Les équivalents de dose efficace dus au retraitement et au stockage des déchets ne sont pas comptabilisés dans ce bilan compte tenu de leurs faibles valeurs, de l'incertitude qui les accompagne, ainsi que de l'époque à laquelle ils seront susceptibles d'être délivrés. Pour le stockage des déchets de haute activité, l'engagement d'équivalent de dose efficace collectif total, c'est-à-dire intégré jusqu'à l'infini serait de 50 h.Sv. [GWe.an] ${ }^{-1}$, d'après l'UNSCEAR [8], avec un maximum annuel de 0,01 h.Sv. [GWe.an] ${ }^{-1}$, et ceci 200000 ans après la fermeture du site, d'après l'étude du KBS [5]. 
Si on retraite tout le combustible, les engagements d'équivalents de dose efficace reçus à l'échelle locale et régionale s'élèvent à 15 h.Sv. [GWe.an] ${ }^{-1}$. Dans ces conditions, la contribution la plus importante en terme de dose est celle du retraitement $(80 \%)$. L'extraction du minerai intervient pour $13 \%$ du total. Le site réacteur intervient pour $5 \%$ du total.

A l'heure actuelle, $5 \%$ du combustible irradié dans le monde est retraité. Pour établir l'engagement d'équivalent de dose efficace moyen dû à l'ensemble de la production électronucléaire mondiale, il y a lieu de pondérer la contribution du retraitement par la fraction effectivement retraitée. Dans ce cas, l'engagement d'équivalent de dose efficace n'est plus que de 4 h.Sv. [GWe.an] ${ }^{-1}$ pour l'ensemble du cycle du combustible, auxquels il conviendrait d'ajouter les doses dues au stockage du combustible non retraité qui ne sont pas connues à l'heure actuelle.

\section{CYCLE DU COMBUSTIBLE NUCLÉAIRE POUR PRODUIRE DE L'ÉLECTRICITÉ PAR FUSION}

Une seule réaction de fusion semble actuellement envisageable à une échelle industrielle : la fusion du deutérium et du tritium.

$$
{ }^{3} \mathrm{H}+{ }^{2} \mathrm{H} \rightarrow{ }^{4} \mathrm{He}(3,5 \mathrm{MeV})+1 \mathrm{n}(14,1 \mathrm{MeV})
$$

\section{a. Opérations sur le combustible et inventaire}

Le cycle du combustible radioactif ne concerne que le tritium; le deutérium ne présentant aucun risque radioactif n'est pas pris en compte ici.

Avec une:première charge de tritium de l'ordre de $500 \mathrm{~g}$ et de deutérium amenée sur le site réacteur, il est possible d'allumer le premier plasma. L'injection du combustible (tritium-deutérium) se fait en deux étapes. Au démarrage, une bouffée de gaz en mélange équimoléculaire de tritium et de deutérium est lâchée dans la chambre torique du Tokamak. Les circuits électriques alimentent alors brutalement les bobines qui génèrent un courant induit toroïdal au milieu de la chambre. Ce courant induit est suffisamment intense pour ioniser complètement le tritium et le deutérium afin de produire un plasma. C'est alors que la deuxième injection de tritium et de deutérium s'effectue sous la forme de petits glaçons lancés à une vitesse de $2 \mathrm{~km} / \mathrm{s}$ de façon à accélérer les particules du plasma et ainsi de chauffer davantage encore le plasma. Ce plasma va brûler quelques minutes en arrosant de neutrons l'ensemble de la couverture et de la première paroi. A l'intérieur de cette première paroi, des circuits d'eau refroidiront le tore et alimenteront en vapeur des turbines; des circuits de lithium plomb (ou d'autres produits selon l'option retenue) produiront du tritium au contact des neutrons (lithium + neutrons $\rightarrow$ tritium). Le plasma s'éteint ensuite et des pompes aspirent les $95 \%$ du tritium injecté non brûlé, le deutérium restant, l'hélium produit par la fusion et éventuellement les produits d'érosion de la paroi du 
tore. Le tritium et le deutérium doivent alors être purifiés et préparés pour une nouvelle injection dans la chambre torique. Le réacteur fonctionnera par pulses de l'ordre de : 1000 s d'ignition, 100 s d'arrêt [6].

En conclusion, sur le site du réacteur, le tritium devra être produit dans la couverture du tore et extrait, il devra être récupéré dans les cendres, et préparé pour l'injection. Une hypothèse raisonnable de $5 \%$ de tritium brûlé par pulse implique que, pour un réacteur de $1 \mathrm{GWe}$, $7,5 \mathrm{~kg}$ de tritium soient aspirés journellement de la chambre et traversent le circuit de retraitement des gaz brûlés. La consommation journalière d'un tel réacteur serait de $420 \mathrm{~g}$ de tritium. La production de tritium dans la couverture serait du même ordre de grandeur [6].

Actuellement, il existe trois projets de Tokamaks impliquant la France : NET de 240 MWe (Next European torus : projet communautaire), INTOR de $200 \mathrm{MWe}$ (International Tokamak reactor: projet AlEA regroupant l'Europe, le Japon, l'URSS, et les USA) et FCTR de 1 GWe (First commercial Tokamak reactor). Le projet NET qui fait suite au projet JET (Joint European torus, construit en Grande Bretagne), concerne plus particulièrement l'étude de la couverture intérieure du tore et le test de différents modes de production du tritium dans la première paroi. L'énergie produite de $740 \mathrm{MWth}$ (soit $240 \mathrm{MWe}$ ) ne serait pas encore récupérée.

L'inventaire total sur le site du Tokamak NET est de $1630 \mathrm{~g}$ à $3630 \mathrm{~g}$ de tritium suivant les auteurs allemands [4] ou français [2]. Sur le site d'un réacteur de 1 GWe.an, il n'y aurait pas 4, 2 fois plus de tritium; en effet, un plasma plus énergétique ne demande pas nécessairement un volume du tore plus grand, mais la quantité du combustible peut tout de même augmenter. L'inventaire devrait être légèrement plus grand tout en restant du même ordre de grandeur.

\section{b. Les rejets}

Les rejets pour la production de la première charge de tritium qui a lieu une fois dans la vie du réacteur devraient être de l'ordre de 18 à $1800.10^{12} \mathrm{~Bq}$ (soit 0,05 à $5 \mathrm{~g}$ ) de tritium. L'ensemble des rejets est présenté sur le tableau III pour les trois projets de Tokamaks NET, INTOR, FCTR.

Pour le réacteur, les sources de produits radioactifs les plus importantes proviennent des opérations où il faut ouvrir le tore, pour le changement de modules de couverture par exemple. Les produits d'érosion du tore posent un problème non négligeable car ils existent en quantité importante. Ils viennent des interactions du plasma sur les matériaux existant au voisinage (couverture). Les particules ainsi formées sont très fines; ces poussières seraient constituées principalement d'isotopes d'hydrogène et de carbone avec une faible proportion d'acier: ${ }^{3} \mathrm{H}(\mathrm{T}),{ }^{4} \mathrm{C},{ }^{57} \mathrm{Co},{ }^{58} \mathrm{Co},{ }^{60} 60,{ }^{58 \mathrm{~m}} \mathrm{Co},{ }^{51} \mathrm{Cr},{ }^{55} \mathrm{Fe},{ }^{59} \mathrm{Fe}, \mathrm{Ni}$, ${ }^{54} \mathrm{Mn},{ }^{99} \mathrm{Mo},{ }^{99 \mathrm{~m}} \mathrm{Tc},{ }^{49} \mathrm{~V},{ }^{181} \mathrm{~W},{ }^{185} \mathrm{~W},{ }^{184} \mathrm{~W},{ }^{182} \mathrm{Ta},{ }^{184} \mathrm{Re}$, avec une activité totale de 0,1 à $0,4 \mathrm{TBq} / \mathrm{g}$ de poussière hors ${ }^{14} \mathrm{C}$ et ${ }^{3} \mathrm{H}$ [7]. 
TABLEAU III

Estimation des rejets gazeux et liquides des différentes phases du cycle du combustible fusion pour les 3 projets NET, INTOR et FCTR en routine avec maintenance (GBq/an)

\begin{tabular}{|c|c|c|c|c|}
\hline $42, n$ & Produfits & 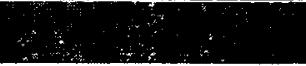 & Rejets & \\
\hline $\begin{array}{l}\text { Production de } \\
\text { la première charge }\end{array}$ & Tritium & & 8000 à 180000 & \\
\hline $\begin{array}{l}\text { Site réacteur } \\
\text { suivant les projets }\end{array}$ & & $\begin{array}{c}\text { NET } \\
\text { (240 MWE/an) }\end{array}$ & $\begin{array}{c}\text { INTOR } \\
(200 \mathrm{MWe} / \mathrm{an})\end{array}$ & $\begin{array}{c}\text { FCTR } \\
\text { (1 GWe/an) }\end{array}$ \\
\hline Rejets gazeux : & 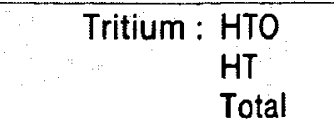 & $<270000$ & $\begin{array}{l}274000 \\
252000 \\
526000\end{array}$ & $\begin{array}{l}444000 \\
333000 \\
777000\end{array}$ \\
\hline & $\begin{array}{l}\text { Produits d'activation } \\
\text { et de corrosion : } \\
\qquad \begin{array}{l}\text { Cr } 51 \\
\text { Mn } 54 \\
\text { Fe } 55 \\
\text { Fe } 59 \\
\text { Co } 58 \\
\text { Co } 60\end{array}\end{array}$ & & $\begin{array}{l}10 \\
5,6 \\
63 \\
0,16 \\
7 \\
2,6\end{array}$ & \\
\hline & Produits d'érosion : & 100 à 6000 & $?$ & $?$ \\
\hline Liquides : & Tritium : HTO & $180000 \AA \grave{A} 600000$ & 37000 & 55500 \\
\hline & $\begin{array}{l}\text { Produits d'activation } \\
\text { et de corrosion : } \\
\qquad \begin{array}{l}\text { Cr } 51 \\
\text { Mn } 54 \\
\text { Fe } 55 \\
\text { Fe } 59 \\
\text { Co } 58 \\
\text { Co } 60\end{array}\end{array}$ & & $\begin{array}{l}10 \\
5,6 \\
63 \\
0,16 \\
7 \\
2,6\end{array}$ & \\
\hline & Produits d'érosion : & 0 & 0 & 0 \\
\hline
\end{tabular}

Le réacteur devrait rejeter au total en tritium 777 TBq. [GWe.an] ${ }^{-1}$ dans l'atmosphère, $60 \%$ sous forme de gaz $H T$ ou $T_{2}$ et $40 \%$ sous forme d'eau HTO. Dans l'eau, il devrait y avoir de l'ordre de 55 TBq. [GWe.an] ${ }^{-1}$ de HTO. Pour les autres produits radioactifs, l'incertitude est plus grande, mais il est possible d'avancer les chiffres suivants: la quantité de produits d'érosion rejetée pourrait atteindre 0,1 à $25 \mathrm{TBq}$.[GWe.an] ${ }^{-1}$ dans l'atmosphère; les produits de corrosion et d'activation rejetés seraient de l'ordre de $20 \mathrm{GBq}$ [GWe.an] ${ }^{-1}$ dans l'atmosphère et de $200 \mathrm{Gbq}[\mathrm{GWe} . \mathrm{an}]^{-1}$ dans la rivière ou la mer.

\section{c. Les déchets}

La présentation des résultats obtenus nécessite certaines précautions en raison des variations encore très importantes que l'on peut observer dans les conceptions successives du projet du futur réacteur. Les déchets sont presque uniquement concentrés sur le site réacteur et viennent de l'activation des structures et non pas du combustible. 
II y aurait 15 à $78 \mathrm{~m}^{3}$ de déchets haute activité par an qui resteraient haute activité pendant 100 ans; au-delà, la décroissance du cobalt 60 étant suffisamment avancée, on peut considérer que la moitié de ces déchets pourrait être classée en déchets de faible activité.

Les activités de ces déchets à l'arrêt du réacteur de fusion seront de l'ordre de 20 à $300000 \mathrm{GBq} / \mathrm{kg}$, ce qui représente, pour $1 \mathrm{GWe}$.an, un total de $10^{7}$ à $3.10^{7} \mathrm{TBq}$ de déchets pour la fusion.

\section{d. Les doses}

La production de $1 \mathrm{GWe}$.an nécessite une fois dans la vie du réacteur de produire et de transporter $500 \mathrm{~g}$ de tritium qui conduisent à un engagement d'équivalent de dose efficace individuel maximal compris entre 0,07 et $30 \mu \mathrm{Sv}$. L'engagement d'équivalent de dose efficace collectif pour la population sera compris entre $10^{-3}$ et 2 h.Sv, c'est-à-dire que si la durée de vie d'un Tokamak est de 20 ans, la contribution annuelle de cette production sera le vingtième de ces doses.

\section{TABLEAU IV}

Bilan concernant la production de l'énergie par fusion pour 1 GWe.an

\begin{tabular}{|c|c|c|c|}
\hline $\begin{array}{l}\text { Gycte du } \\
\text { combustrole }\end{array}$ & Thuentaline & Rejets & 7rose publis \\
\hline $\begin{array}{l}\text { Production } \\
\text { de la } 1^{\text {ere }} \\
\text { charge }\end{array}$ & $\begin{array}{l}\text { tritium } \\
500 \mathrm{~g}\end{array}$ & $\begin{array}{l}\text { tritium } \\
20000 \text { à } \\
2000000 \mathrm{GBq}\end{array}$ & $10^{-3}$ à 2 h.Sv/Tokamak \\
\hline $\begin{array}{l}\text { Site du } \\
\text { réacteur }\end{array}$ & $\begin{array}{l}\text { tritium } \\
3 \text { à } 5 \mathrm{~kg}\end{array}$ & $830000 \mathrm{GBq} / \mathrm{an}$ & $\begin{array}{l}\text { gaz : } 0,4 \text { à } 0,8 \text { h.Sv/an } \\
\text { liquide : } 0.2 \text { à } 0,8 \text { h.Sv/an }\end{array}$ \\
\hline $\begin{array}{l}\text { Stockage } \\
\text { des déchets }\end{array}$ & $\begin{array}{l}\text { haute activité } \\
15 \text { à } 78 \mathrm{~m}^{3}\end{array}$ & & ? \\
\hline
\end{tabular}

Le reste du cycle du combustible se passe sur le site réacteur sauf pour le stockage des déchets. Un réacteur de fusion conduit à un engagement d'équivalent de dose efficace délivré au public, pour les rejets en tritium dans l'atmosphère, de l'ordre de 1 à $10 \mu \mathrm{Sv}$. [GWe.an] ${ }^{-1}$ pour l'individu du public le plus exposé et l'engagement d'équivalent de dose efficace collectif sera de 0,4 à 0,8 h.Sv. [GWe.an] ${ }^{-1}$ selon le site pour la population résidant dans les $900 \mathrm{~km}$ autour du site. Ces valeurs ont été établies par analogie avec les calculs de dose effectués pour trois sites français [7]. Les rejets en tritium dans les effluents liquides impliquent un engagement d'équivalent de dose efficace individuel maximal de 0,02 à $0,2 \mu \mathrm{Sv}$. [GWe.an] ${ }^{-1}$, et un engagement d'équivalent de dose efficace collectif de 0,2 à 0,8 h.Sv. [GWe.an] ${ }^{-1}$ selon le site retenu [7].

Le stockage des déchets haute activité du NET $\left(24 \mathrm{~m}^{3}\right.$ de déchets) représente un équivalent de dose efficace maximal individuel de 0,2 Sv/an pour un stockage terrestre et $2,510^{-7}$ Sv/an pour un stockage immergé [3]. 
Cet équivalent de dose efficace est uniquement dû au tritium et n'inclut pas le traitement des déchets, les produits d'activation, de corrosion et d'érosion qui sont relachés en quantité beaucoup plus faible que le tritium. Les doses dues aux produits radioactifs autres que le tritium n'ont pas pu'être évaluées par manque de données précises, entre autres celles sur les produits d'érosion. Cependant, les engagements d'équivalents de dose efficace collectifs dus aux radionucléides autres que le tritium devraient être, d'après les experts de la fusion, moins importants que ceux dus au tritium. Mais le calcul reste à faire car ils ne devraient pas être négligeables pour autant.

\section{CONCLUSION}

Le tableau $V$ réalise une synthèse permettant la comparaison de la production de 1 GWe.an par un REP ou par un RF.

\section{TABLEAU $V$}

Comparaison finale pour les deux cycles du combustible fission et fusion sur : les inventaires, les rejets, les déchets et les équivalents de dose efficace pour une production de 1 GWe.an

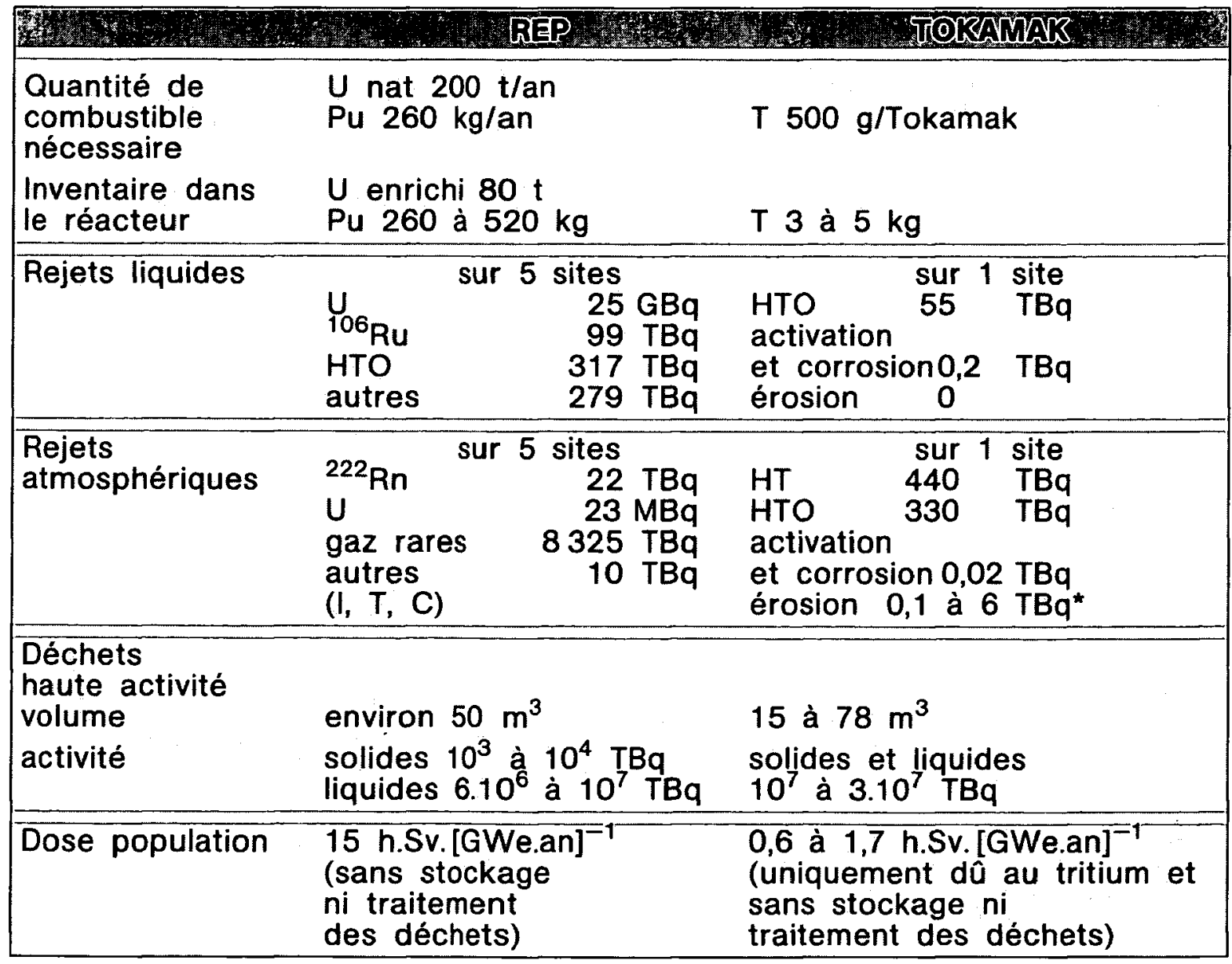

* Extrapolation tirée du réacteur JET. 
Au niveau de l'inventaire de produit combustible, un Tokamak présente l'énorme avantage de ne mettre en jeu qu'une petite quantité de produit à période courte par rapport aux grandes quantités à période radioactive nettement plus longue nécessaires au fonctionnement d'un REP. Pour produire 1 GWe.an avec un REP, 200 t d'uranium naturel sont nécessaires auxquelles viennent s'ajouter, dans les réacteurs, une masse de $260 \mathrm{~kg}$ de plutonium, résidu de ${ }^{238} \mathrm{U}$ mais aussi utilisée en partie comme combustible. L'activité ainsi mise en jeu est de 2,6 TBq pour l'uranium naturel pour une période de $10^{9}$ ans et $1,3.10^{5} \mathrm{TBq}$ pour le plutonium dont la période varie de 15 ans à $3,8.10^{5}$ ans selon l'isotope considéré. Les 3 à $5 \mathrm{~kg}$ de tritium présent dans un Tokamak correspondent à une activité de $10^{6}$ à $210^{6} \mathrm{TBq}$.

L'activité rejetée dans l'atmosphère et les eaux de surface par la production de 1 GWe.an par un REP est de l'ordre de $27 \mathrm{GBq}$ d'uranium et $22 \mathrm{TBq}$ de radon, les autres produits viennent principalement du retraitement du combustible avec $262 \mathrm{TBq}$ de tritium, $210 \mathrm{TBq}$ d'émetteurs bêta, et $90 \mathrm{GBq}$ d'émetteurs alpha. Pour la filière fusion, une première approche indique que l'activité rejetée pour la même production serait de l'ordre de $830 \mathrm{TBq}$ de tritium. Le stockage pour les deux filières n'est pas comptabilisé.

Les engagements d'équivalents de dose efficace délivrés à la population sont directement liés aux rejets. Pour le fonctionnement normal et la maintenance, ils s'élèvent, pour l'ensemble du cycle du combustible correspondant aux REP, à 15 h.Sv. [GWe.an] ${ }^{-1}$, sans compter les terrils ou le stockage des déchets, dans le cas où $100 \%$ du combustible est retraité. Actuellement, seuls $5 \%$ du combustible irradié étant retraité, l'équivalent de dose efficace collectif n'est que de 4 h.Sv. [GWe.an] ${ }^{-1}$ pour l'ensemble du cycle du combustible, sans tenir compte du stockage du combustible irradié non traité qui reste inconnu.

Le cycle du combustible "fusion" conduit à un équivalent de dose efficace collectif délivré au public de l'ordre de 0,6 à 1,7 h.Sv. [GWe.an] ${ }^{-1}$, en intégrant la production de la première charge. Cet engagement d'équivalent de dose efficace collectif est uniquement dû au tritium et n'inclut pas le traitement des déchets, leur stockage, les produits d'activation, de corrosion et d'érosion qui sont relâchés en quantité beaucoup plus faible que le tritium. L'importance de ce qui est négligé devrait entraîner des engagements d'équivalent de dose efficace moindre que ceux dus au tritium, et c'est pourquoi seul le tritium est considéré dans cette première étude. Mais l'évaluation reste à faire, principalement sur les produits d'érosion qui ne sont, toutefois, -probablement pas négligeables.

Si les déchets de haute activité sont apparemment de volume et d'activité identiques pour les deux filières, il faut insister sur le fait que les déchets de la fusion sont nettement différents de ceux de la fission en ce qui concerne la durée de vie du radionucléide à vie courte prépondérant. En effet, la décroissance du césium 137 des éléments combustibles de REP nécessite au moins 300 ans alors que la décroissance du cobalt 60 des déchets de la fusion ne demande que 70 ans. 
Pour l'ensemble du cycle de la fusion, il y aurait 15 à $78 \mathrm{~m}^{3}$ de déchets haute activité par an qui resteraient haute activité pendant 100 ans; au-delà, la décroissance du cobalt 60 étant suffisamment avancée, on peut considérer que la moitié de ces déchets pourrait être classée en déchets de faible activité. Comparativement, un REP produit $11 \mathrm{~m}^{3} / \mathrm{an}$ de déchets haute activité pour plus de 300 ans; et le retraitement du combustible d'un REP entraîne 36 à $56 \mathrm{~m}^{3} /$ an de déchets haute activité qui resteraient haute activité pendant au moins 300 ans.

En conclusion, cette nouvelle filière ne serait donc pas aussi négative qu'elle le laissait paraître avec sa production de déchets haute activité ; ces chiffres présentent même un avantage sur le plan de la radioprotection. Si cette filière paraît intéressante de ce point de vue, elle n'écarte pas, pour autant, tous les problèmes traditionnels du cycle du combustible nucléaire qui sont de manipuler, de traiter des produits radioactifs, d'évacuer les rejets vers l'atmosphère ou les eaux de surface, de stocker des déchets radioactifs à court et à long terme. Ces problèmes seront généralement moindres que ceux créés par la filière fission, sauf dans le cas du traitement des déchets haute activité où des pièces de plusieurs centaines de tonnes (segments du tore) fortement activées par du tritium et d'autres produits irradiants devront être déplacées, découpées et/ou fondues immédiatement à l'arrêt du réacteur.

II resterait maintenant à comparer les expositions professionnelles et les expositions probabilistes dues à un éventuel accident qui n'ont pas été introduites dans le cadre de cette étude.

\section{REMERCIEMENTS}

Cet article a bénéficié d'un financement en provenance des Communautés européennes Contrat SE.7.

\section{RÉFÉRENCES}

[1] COULON R., AIGUEPERSE J., ANGUENOT F. - Etude comparative sous les deux aspects radioactif et chimique de l'impact sur la population des industries conventionnelles et nucléaires. Programme Grand Delta, Rapport 1987. Fontenay-aux-Roses : CEA/IPSN/DPS, 1987.

[2] DJERASSI H., LEGER D., ROUILLARD J. - NET - OPAS Section 3.7 - Tritium and fuel handling system, final report. Rapport CEA/IPSN/DPT/ SE 5.4/88.05 (Sûreté et environnement), Août 1988.

[3] GUETAT Ph. - Fusion reactor wastes. Technical and radiological aspects for the management of wastes from NET and commercial reactor. AIEA Comité technique sur la sûreté des réacteurs à fusion, Culham (Grande Bretagne), 3-7 novembre 1986. In : AIEA. Fusion reactor safety. Rapport IAEA-TECDOC-440, 1987.

[4] GULDEN W., RAEDER J. - Safety aspects of the Next european Torus. Fusion Technol., 1988, 14, 218-227. 
[5] KAERNBRAENSLESAEKERHET (KBS), Stockholm - Handling of spent nuclear fuel and final storage of vitrified high level reprocessing waste. Rapport suédois KBS, 1979 (traduction anglaise INIS - mf - 5909).

[6] LEGER D., ROUYER J.L. - Le tritium et les réacteurs à fusion contrôlée. In : Journées Tritium, Dijon, 23-25 avril 1986. Fontenay-aux-Roses : Société Française de radioprotection, 1986, 27-49.

[7] RANCILLAC F., DESPRES A. - Comparaison de l'impact radiologique de la production d'énergie par des réactions de fusion et des réactions de fission. Rapport DPS/SEGP/LSEES - 88/18 (existe aussi en anglais), 1988.

[8] UNITED NATIONS SCIENTIFIC COMMITTEE ON THE EFFECTS OF ATOMIC RADIATION (UNSCEAR) - Sources, effects and risks of ionizing radiation 1988, report to the general assembly, with annexes (Sales $n^{\circ}$ E.88.IX.7). Genève : Nations Unies, 1988. 